\title{
Erratum to: Thermodynamically Consistent Limiting Forced Convection Heat Transfer in a Asymmetrically Heated Porous Channel: An Analytical Study
}

\author{
P. K. Mondal
}

Published online: 10 December 2013

C) Springer Science+Business Media Dordrecht 2013

\section{Erratum to: Transp Porous Med (2013) 100:17-37 DOI 10.1007/s11242-013-0203-5}

In our recent paper (P. K. Mondal, 2013; Transp Porous Med (2013) 100:17-37), Section 2 contains the closed form expression of temperature profile (Eq. 16) and expressions of different terms like $I_{1}-I_{11}$ are incorrect in the form in which it appears. The expression of temperature profile along with each of the expression of $I_{1}, I_{6}$ and $I_{11}$ contains typographical error. In this erratum we seek to rectify the errors, and present those in a corrected form as given below. However, rest of the formulations and results are correct.

$$
\begin{aligned}
\theta= & \frac{(1-A)}{(1+A)} \hat{y}+Q\left[\frac{1}{2}-\frac{\hat{y}^{2}}{2}\right]+\frac{\mathrm{Br}}{R_{1} \cdot \mathrm{Da}}\left[\begin{array}{l}
\left\{\frac{1}{2}-\frac{\hat{y}^{2}}{2}\right\}+\frac{1}{2 \cosh ^{2} \beta}\left\{\frac{1}{2}-\frac{\hat{y}^{2}}{2}\right\} \\
+\frac{1}{4 \beta^{2} \cosh ^{2} \beta}\left\{\frac{\cosh (2 \beta)}{2}-\frac{\cosh (2 \beta) \hat{y}}{2}\right\} \\
-\frac{2}{\beta^{2}}\left\{1-\frac{\cosh \beta \hat{y}}{\cosh \}}\right.
\end{array}\right] \\
& +\frac{R_{2}}{2 \varepsilon}\left[\left\{\frac{1}{2}-\frac{\hat{y}^{2}}{2}\right\}-\frac{1}{2 \beta^{2}}\left\{\frac{\cosh (2 \beta)}{2}-\frac{\cosh (2 \beta) \hat{y}}{2}\right\}\right] \\
I_{1}= & \frac{S}{R_{1}^{1 / 2}[0]} \\
I_{6}= & \frac{\mathrm{Br}}{8 R_{1}^{3 / 2} \cdot \mathrm{Da} \cdot \beta^{2} \cdot \cosh ^{2} \beta}\left[\frac{\sinh (2 \beta)}{\beta}-\left(\frac{3 \sinh \beta+\sinh (3 \beta)}{3 \beta \cosh \beta}\right)\right] \\
I_{11}= & \frac{R_{2}}{8 \varepsilon \cdot \beta^{2} \cdot R_{1}^{1 / 2}}\left[\frac{\sinh (2 \beta)}{\beta}-\left(\frac{3 \sinh \beta+\sinh (3 \beta)}{3 \beta \cosh \beta}\right)\right]
\end{aligned}
$$

The online version of the original article can be found under doi:10.1007/s11242-013-0203-5.

P. K. Mondal $(\bowtie)$

Department of Mechanical Engineering, Kalyani Government Engineering College,

Nadia, Kalyani 741235, India

e-mail: pranab2k3@yahoo.com 